\title{
A symmetry reduction method for continuum structural topology optimization
}

\author{
Iku Kosaka ${ }^{\mathrm{a}, 1}$, Colby C. Swan ${ }^{\mathrm{a}, \mathrm{b}}$ \\ a Department of Civil and Environmental Engineering, The University of Iowa, Iowa City, IA 52242, USA \\ ${ }^{\mathrm{b}}$ Center for Computer Aided Design, The University of Iowa, Iowa City, IA 52242, USA
}

\begin{abstract}
It is considered that asymmetrical material layout design solutions are caused by numerical roundoff and the convexity characteristics of alternative topology design formulations. Emphasis is placed here not on analyzing potential instabilities that lead to asymmetrical designs, but on a method to stabilize topology design formulations. A novel symmetry reduction method is proposed, implemented and studied. While enforcing symmetry and significantly reducing the size of the optimization problem, the symmetry reduction method is shown to have the added benefit of greatly simplified design sensitivity analysis of non-simple repeated vibrational eigenvalues which occur in many symmetrical structures. (C) 1998 Elsevier Science Ltd. All rights reserved.
\end{abstract}

Keywords: Topology; Topology optimization; Continuum topology; Design sensitivity analysis (DSA); Optimal design; Symmetry enforcement; Structural optimization

\section{Introduction and motivation}

Continuum structural topology optimization is an increasingly powerful design tool which can be used to optimize the material arrangements in structural systems to achieve a wide variety of performance objectives, including as examples: minimal compliance [1-4]; optimal strength [5,6]; viscoelastic damping [7]; and compliant mechanisms [8-10]. For all of these structural or material topology design optimization applications, if the design domain, the boundary conditions and the loading are all symmetrical, then the optimum design solution is also expected to be symmetrical. In practice, however, the design solutions obtained may not feature the expected symmetries: (1) due to numerical roundoff errors in the design sensitivity analysis and optimization procedures; and (2) if the optimiz-

\footnotetext{
${ }^{1}$ Now with VMA Engineering, Colorado Springs, Colorado, USA
}

ation problem formulation is not strictly convex giving rise to the existence of locally optimal asymmetrical design solutions. Since numerical roundoff is always present irrespective of the problem formulation, the issue of whether or not symmetrical design solutions are obtained is highly dependent upon the problem formulation. In this paper, the term 'problem formulation' refers to the manner in which the continuous topology optimization problem is posed and developed.

In continuum structural topology optimization, various problem formulations exist, and they can be broadly categorized into two general classes of methods: (1) relaxed formulations involving mixtures of assumed parameterized micro-morphologies; and (2) continuous formulations which use mixing rules that assume and involve no microstructure. Characteristic of the problem formulations which assume micro-morphologies of the mixture, are the homogenizationbased porous solid formulation $[1,11]$ and the rank-2 plane stress laminate formulation [2]. From the 
examples presented in the literature, these problem formulations appear to be somewhat, although not completely, stable with respect to symmetry of the designs at least for compliance minimization problems. For other classes of design objectives such as viscoelastic damping and compliant mechanisms, these formulations have not yet been shown to be convex and could thus be prone to achieving spatially asymmetrical designs.

On the other hand, continuum structural topology optimization formulations which assume no microstructure, including the simple density based power law mixing rule [3,12-14] and the classical hybrid VoigtReuss mixing rule [4,5], can show a wide range of different characteristics. For elastic compliance minimization problems, topology optimization formulations that use the very stiff Voigt mixing rule have been shown to be convex [4] and thus very stable with respect to symmetry, but generally realize solutions that are neither interpretable nor manufacturable. For the design of structures for such performance objectives as high viscoelastic damping [7], or development of efficient compliant mechanisms [10], however, even topology formulations based on stiff mixing rules have been found to be lacking strict convexity so that a wide variety of locally optimal solutions exist, some of which are typically asymmetrical. On the other hand, topology formulations that use very compliant mixing rules, such as the Reuss formulation or highly penalized density formulations, generally achieve designs which are highly interpretable and manufacturable, but have been shown to be potentially nonconvex for elastic compliance minimization and other classes of problems. Topology formulations based on compliant mixing rules are therefore prone to achieving asymmetrical material layout designs.

The motivation here is, therefore, to briefly present a design space reduction method for stabilization of topology design formulations with respect to symmetry so that undesirable asymmetrical local optimum solutions can be avoided. The proposed method will be shown to achieve meaningful spatially symmetrical designs even for asymmetrical loadings and even when mesh discretizations themselves are not themselves precisely symmetrical. In Ref. [15] the design space reduction method was compared with the multiple loading condition symmetry enforcement method investigated in [16]. While both methods should in the absence of numerical roundoff produce identical solutions, the design space reduction method developed here is more effective and efficient.

Another favorable attribute of the proposed symmetry reduction method is that it facilitates design sensitivity analysis of vibrational eigenvalues. Repeated eigenvalues often occur in optimized, symmetrical structures, and computational difficulties arise when computing their design sensitivities due to the fact that they are not generally differentiable but only directionally differentiable [17]. Methods for solving optimization problems involving nondifferentiable repeated eigenvalues have been investigated in Ref. [18-20]. Sizing optimization problems to maximize the buckling capacity of structures have also been found to frequently encounter repeated buckling load eigenvalues and modes that are not simply associated with the symmetry of structures [21]. In [22] the physical origin and ambiguity of repeated eigenvalues were discussed, and a treatment was presented to obtain partial derivatives of repeated eigenvalues. In Ref. [23] it was shown that, under special circumstances, the treatment of Ref. [22] is not necessary to obtain the partial derivatives of repeated eigenvalues.

With reference to these preceding works, it will be shown that with the proposed symmetry reduction methods, those repeated eigenvalues strictly associated with the symmetry of the structure are indeed differentiable, and thus the special treatments suggested in Refs $[19,20]$ are not always necessary. Therefore, the proposed symmetry reduction method is potentially attractive for solving vibrational eigenvalue optimization problems since it permits usage of identical design sensitivity expressions for both simple and some non-simple eigenvalues, while reducing the size of the optimization problem and maintaining symmetrical structures.

In this paper, the proposed symmetry reduction algorithm is implemented and studied in the context of microstructure free topology formulations. The symmetry reduction method can also be extended to relaxed topology formulations based on parameterized micro-morphologies, although the implementation would be more involved. The remainder of this paper is organized as follows: in Section 2, a Voigt-Reuss continuum structural topology optimization framework is briefly reviewed. The proposed symmetry reduction method is then presented and its characteristics are discussed in Section 3 in the context of two representative structural topology optimization problems. In Section 4 , it is further demonstrated that the proposed symmetry reduction method has the additional benefit of eliminating the computational difficulties that can arise computing design sensitivities of repeated eigenvalues which are frequently encountered in symmetrical structural systems being optimized. Then, an example topology design optimization problem is solved for a square, flat plate whose first five vibrational eigenvalues, which include a non-simple repeated root, are maximized. In concluding, brief remarks on the proposed method are made in Section 5. 


\section{Voigt-Reuss continuum structural topology formulation}

\subsection{Distribution of materials}

The complete undeformed spatial domain of the structure being designed is denoted by $\Omega_{\mathrm{S}}$; its designable subset by $\Omega_{\mathrm{D}}$; and its non-designable subset, in which the spatial/topological arrangement of materials is taken to be fixed, by $\Omega_{\mathrm{N}}$. The arrangement of two pre-selected candidate materials $\mathscr{A}$ and $\mathscr{B}$ in $\Omega_{\mathrm{D}}$ remains to be determined and so this region is called designable. A set of single or multiple loading/boundary conditions to which $\Omega_{\mathrm{S}}$ will be subjected are specified and a starting design $\mathbf{b}^{(0)}$ which specifies the initial material layout in $\Omega_{\mathrm{D}}$ is selected. For each set of loading/boundary conditions, the structure is analyzed as a boundary value problem and/or an eigenvalue problem. The objective of the variable topology material layout design process is to iteratively improve upon the initial design of the structure (that is the spatial arrangement of the two candidate materials in $\Omega_{\mathrm{D}}$ ) until an optimal design is achieved. Accordingly, an objective functional which measures the desired behavior of the structure must be specified, along with constraint functionals which place restrictions on the design, and side constraints which place explicit bounds on the values that can be taken by the individual design variables.

Preference is given to discrete final material distributions that satisfy $\Omega_{\mathscr{A}} \cap \Omega_{\mathscr{B}}=\emptyset$ and $\Omega_{\mathscr{A}} \cup \Omega_{\mathscr{R}}=\Omega_{\mathrm{S}}$, where $\Omega_{\mathscr{A}}$ is the spatial domain occupied by material $\mathscr{A}$ and $\Omega_{\mathscr{B}}$ is the spatial domain occupied by material $\mathscr{B}$. These distributions are achieved, however, through continuous formulations which permit mixtures to exist throughout the design domain $\Omega_{\mathrm{D}}$, at least in intermediate design states. By permitting mixtures, the material phases $\mathscr{A}$ and $\mathscr{B}$ are allowed to simultaneously and partially occupy an infinitesimal neighborhood about each spatial point $\mathbf{X}$ in $\Omega_{\mathrm{D}}$. Such mixtures can be described using volume fraction or density ideas. For example, the volume fraction of material phase $\mathscr{A}$ at a fixed spatial point $\mathbf{X}$ in the design domain $\Omega_{\mathrm{D}}$ is denoted by $\phi_{\mathscr{A}}(\mathbf{X})$ and represents the fraction of an infinitesimal volume element surrounding point $\mathbf{X}$ occupied by material $\mathscr{A}$. The volume fraction definition for material phase $\mathscr{B}$ is similar. Natural constraints upon the spatial volume fractions for the twomaterial problem are:

$$
\begin{gathered}
0 \leq \phi_{\mathscr{A}}(\mathbf{X}) \leq 1 ; \quad 0 \leq \phi_{\mathscr{B}}(\mathbf{X}) \leq 1 ; \\
\phi_{\mathscr{A}}(\mathbf{X})+\phi_{\mathscr{B}}(\mathbf{X})=1 .
\end{gathered}
$$

Since the material volume fractions at $\mathbf{X}$ are not independent, in the two-material problems one need only be concerned with the layout of phase $\mathscr{A}$ since that of phase $\mathscr{B}$ follows directly from Eq. (1).

In the proposed topology design optimization framework, the design domain $\Omega_{\mathrm{D}}$ will be discretized into NEL low-order finite elements such as bilinear continuum degenerated shell elements; bilinear planar continuum elements; or trilinear three-dimensional continuum elements. For these low-order elements, the independent material volume fraction $\phi_{\mathscr{A}}$ is taken as piecewise constant over the spatial domain occupied by individual finite elements. The designable spatial/ topological distribution of material phase $\mathscr{A}$ in $\Omega_{\mathrm{D}}$ can thus be described by a vector of design variables $\mathbf{b}$ with contributions from each element comprising $\Omega_{\mathrm{D}}$. Specifically, the design vector $\mathbf{b}$ has the definition:

$\mathbf{b}:=\left\{\phi_{\mathscr{A}_{1}}, \phi_{\mathscr{A}_{2}}, \ldots, \phi_{\mathscr{A}_{\mathrm{NEL}}}\right\}$.

That is, the full vector of design variables $\mathbf{b}$ is comprised of NEL scalar-valued element level contributions $\phi_{\mathscr{A}}$, each of which represents the volume fraction of phase $\mathscr{A}$ in the $i$ th element. This system allows the two candidate materials to be arbitrarily distributed throughout the NEL finite elements comprising the design domain $\Omega_{\mathrm{D}}$, subject only to natural constraints of Eq. (1).

Global material cost constraints are generally imposed upon the designed structure by specifying appropriate upper or lower limits on the global volume fraction of the independent material phase. A typical upper bound for a solid phase is represented as $\left\langle\phi_{\mathscr{A}}\right\rangle-C_{\mathscr{A}} \leq 0$, where $C_{\mathscr{A}}$ is a designer specified upper bound value on the global volume fraction of material phase $\mathscr{A}$ in the structural domain $\Omega_{\mathrm{S}}$. The global volume fraction of phase $\mathscr{A}$ over the structural domain $\Omega_{\mathrm{S}}$ is calculated as:

$\left\langle\phi_{\mathscr{A}}\right\rangle=\frac{\int_{S} \phi_{\mathscr{A}}(\mathbf{X}) \mathrm{d} \Omega_{\mathrm{S}}}{\int_{S} \mathrm{~d} \Omega_{\mathrm{S}} .}$

Remark 2.1. It is recognized that topology optimization formulations that use low-order $C^{0}$ finite elements and design variables that are uniform on element domains are potentially unstable and prone to achieve "checkerboarding solutions' $[24,25]$. This difficulty can easily be solved, however, by utilizing the filtering methods proposed in Ref. $[3,4]$.

\subsection{Constitutive mixing rules}

Since each finite element of the design domain $\Omega_{\mathrm{D}}$ generally contains a mixture of materials $\mathscr{A}$ and $\mathscr{B}$, a method is needed to prescribe the constitutive behavior of such mixtures. In the proposed topology optimization framework, the constitutive behaviors are 


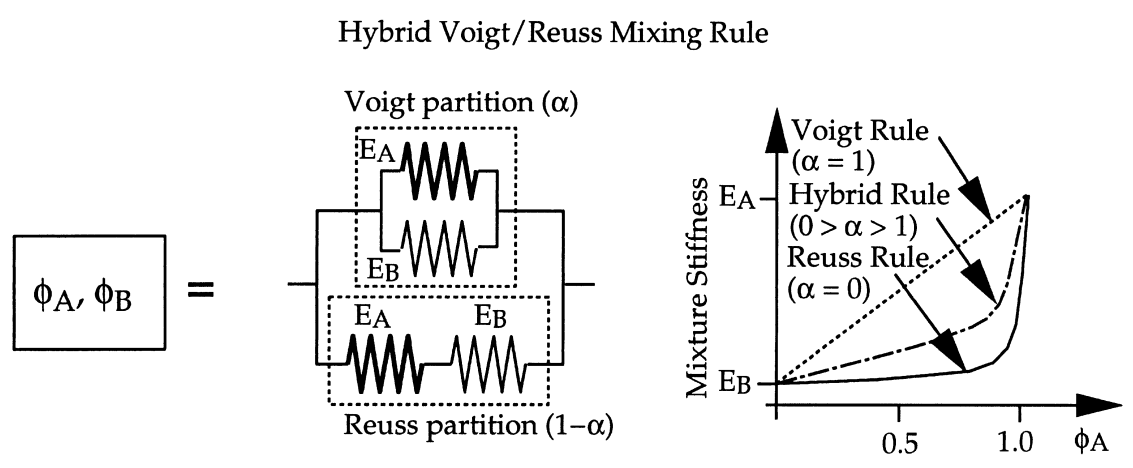

Fig. 1. Schematic of effective stiffness of the hybridized Voigt-Reuss mixtures. For the diagrams shown, material $\mathscr{A}$ is taken to be stiffer than material $\mathscr{B}$.

assumed to be governed by the classical Voigt and Reuss mixing rules, and hybrid combinations thereof (Fig. 1).

For multi-dimensional mixtures, the Voigt rule assumes that the material phases share the same local strain tensor, whereas the Reuss rule assumes that the materials share the same local stress tensor. The associated decomposition equations for the Voigt mixing of two general phases at a given material point $\mathbf{X}$ are:

$\boldsymbol{\epsilon}_{\mathrm{Voigt}}=\boldsymbol{\epsilon}_{\mathscr{A}}=\boldsymbol{\epsilon}_{\mathscr{B}}$

$\boldsymbol{\sigma}_{\text {Voigt }}=\phi_{\mathscr{A}} \boldsymbol{\sigma}_{\mathscr{A}}(\epsilon)+\phi_{\mathscr{B}} \boldsymbol{\sigma}_{\mathscr{B}}(\epsilon)$.

The corresponding decomposition equations for the Reuss mixing of two general materials are:

$\boldsymbol{\epsilon}_{\text {Reuss }}=\phi_{\mathscr{A}} \boldsymbol{\epsilon}_{\mathscr{A}}+\phi_{\mathscr{B}} \boldsymbol{\epsilon}_{\mathscr{B}}$

$\sigma_{\text {Reuss }}=\sigma_{\mathscr{A}}\left(\epsilon_{\mathscr{A}}\right)=\sigma_{\mathscr{B}}\left(\epsilon_{\mathscr{B}}\right)$

For the hybrid Voigt-Reuss mixture (Fig. 1), the assumption is that both branches of the mixture have the same strain and that the volume fraction of the total mixture in the Voigt branch is $\alpha$ and that in the Reuss branch is $1-\alpha$. Accordingly, the effective stresses and strains of the partitioned mixture are:

$\boldsymbol{\epsilon}=\boldsymbol{\epsilon}_{\mathrm{Voigt}}=\boldsymbol{\epsilon}_{\text {Reuss }}$

$\boldsymbol{\sigma}=\alpha \boldsymbol{\sigma}_{\text {Voigt }}+(1-\alpha) \boldsymbol{\sigma}_{\text {Reuss }}$.

Here, it is proposed that $\alpha \in[0,1]$ be treated as a constant, with $\alpha=1$ yielding a pure Voigt mixture and $\alpha=0$ yielding a pure Reuss mixture.

\subsection{Objective and constraint functionals}

Numerous formulation options exist in structural topology design optimization in terms of utilizing assorted combinations of objective and constraint functionals $\mathscr{B}$. The design variables as specified in
Eq. (2) are continuous and real-valued, and it is assumed that dependent functionals, both objective and constraints, will also be continuous, real-valued, and piecewise differentiable.

It is useful to distinguish between those functionals $\mathscr{B}$ which are structural response-independent (that is $\mathscr{B}=\mathscr{B}(\mathbf{b})$ ), and those which are response-dependent (that is $\mathscr{B}=\mathscr{B}(\mathbf{b}, \mathbf{u})$ ), where the performance or state of the structure being designed is described in terms of u, the vector displacement field. An example of a response-independent functional for the structural topology optimization problem is the global volume fraction of one of the candidate material phases, defined as:

$\Phi_{\mathscr{A}}=\left\langle\phi_{\mathscr{A}}\right\rangle-\mathscr{B}_{\mathscr{A}}$

in which $\left\langle\phi_{\mathscr{A}}\right\rangle$ represents the volume average of $\phi_{\mathscr{A}}$ over the entire analysis domain $\Omega_{\mathrm{S}}$. Other responseindependent functionals are for example 'perimeter' metrics [26]; and intermediate volume measures [26], among many others. In contrast, the elastic structural compliance functional for specific loading conditions is response-dependent functional, being defined as:

$2 \Pi=\int_{S} \mathbf{q} \cdot \mathbf{u} \mathrm{d} \Omega_{\mathrm{S}}+\int_{h} \mathbf{h} \cdot \mathbf{u} \mathrm{d}_{\mathrm{h}}-\int_{g} \mathbf{t}^{n} \cdot \mathbf{g} \mathrm{d}_{\mathrm{g}}$,

where $\mathbf{q}, \mathbf{h}$ and $\mathbf{g}$ are the body forces, boundary tractions, and prescribed displacement vectors, respectively, applied to $\Omega_{\mathrm{S}}$. There are a wide variety of other possible response-dependent functionals including: eigenvalues [27]; ultimate strength [5,6]; compliant mechanisms [8-10]; and viscoelastic damping [7], to name a few.

\subsection{The analysis problem}

In this study, topology design is used to find the optimal layout of materials in structures to minimize elastic compliance and to tailor vibrational eigenvalues. 
These types of objectives require the solution of linear elliptic boundary value problems and linear eigenvalue problems which are briefly formulated below.

\subsubsection{Linear quasi-static analysis of structures}

The standard strong form of the linear elliptic boundary value problems for structural analysis is: find $\mathbf{u}: \Omega_{\mathrm{S}} \mapsto \mathfrak{R}^{3}$ such that

$\sigma_{i j, i}+\rho q_{j}=0 \quad$ on $\quad \Omega_{\mathrm{S}}$

subject to the boundary conditions:

$$
\begin{aligned}
u_{j}=g_{j} & \text { on } \quad \Gamma_{g_{j}} \text { for } j=1,2,3 \\
n_{i} \sigma_{i j}=h_{j} & \text { on } \quad \Gamma_{h_{j}} \text { for } j=1,2,3 .
\end{aligned}
$$

For well-posedness, it is assumed that the surface $\Omega$ of the structural domain $\Omega_{\mathrm{S}}$ admits the decomposition $\Gamma=\overline{\Gamma_{g_{j}} \cup \Gamma_{h_{j}}}$ and $\Gamma_{g_{j}} \cap \Gamma_{h_{j}}=\emptyset$, for $j=1,2,3$. The constitutive behavior of the material (or mixture of materials) occupying $\Omega_{\mathrm{S}}$ relates the local stress $\boldsymbol{\sigma}$ to local strain $\boldsymbol{\epsilon}=\frac{1}{2}\left[(\nabla \mathbf{u})+(\nabla \mathbf{u})^{\mathrm{T}}\right]$ through a linear elastic constitutive model of the general form

$\boldsymbol{\sigma}=\mathbf{C}: \boldsymbol{\epsilon}$,

where the $\mathbf{C}$ is the effective elastic constitutive tensor of the local mixture and depends upon: the properties of materials $\mathscr{A}$ and $\mathscr{B}$; the local volume fractions ( $\phi_{\mathscr{A}}$, $\left.\phi_{\mathscr{B}}\right)$; and the mixing rule being employed.

The weak or variational form of the problem is obtained by restating the strong form (9), as

$\int_{S}\left[\sigma_{i j, i} \delta u_{j}+\rho q_{j} \delta u_{j}\right] \mathrm{d} \Omega_{\mathrm{S}}=0$,

from which integration by parts, usage of the divergence theorem and utilization of the natural boundary conditions gives the virtual work equation

$\int_{S} \sigma_{i j} \delta \epsilon_{i j} \mathrm{~d} \Omega_{\mathrm{S}}-\int_{S} \rho q_{j} \delta u_{j} \mathrm{~d} \Omega_{\mathrm{S}}-\int_{\Gamma_{h}} h_{j} \delta u_{j} \mathrm{~d} \Gamma_{h}=0$.

Discretization of the spatial domain $\Omega_{\mathrm{S}}$ into a finite element mesh and usage of a Galerkin formulation in which the real $\mathbf{u}$ and variational $\delta \mathbf{u}$ kinematic fields are expanded in terms of the same nodal basis functions leads to the following force balance equations at each unrestrained node $A$ in the mesh:

$\mathbf{r}_{A}=\mathbf{f}_{A}^{\mathrm{int}}-\mathbf{f}_{A}^{\mathrm{ext}}=\mathbf{o}$

where

$\mathbf{f}_{A}^{\mathrm{int}}=\int_{S} \mathbf{B}_{\mathrm{A}}^{\mathrm{T}}: \boldsymbol{\sigma} \mathrm{d} \Omega_{\mathrm{S}}=\mathbf{K}_{A B} \cdot \mathbf{u}_{B}$

$\mathbf{f}_{A}^{\text {ext }}=\int_{S} \rho N_{A} \mathbf{q} \mathrm{d} \Omega_{\mathrm{S}}+\int_{\Gamma_{h}} N_{A} \mathbf{h} \mathrm{d} \Gamma_{h}$.

For the class of problems being treated here, Eq. (14) represents a set of linear algebraic equations which can be solved in any number of ways. In Eq. (15), $\mathbf{B}$ and $N_{A}$ represent the nodal strain-displacement operator and the nodal shape function associated with isoparametric finite elements.

\subsubsection{Weighted compliance functional}

One method for obtaining optimal material layout designs of minimal compliance for multiple loading and/or restraint conditions on $\Omega_{\mathrm{S}}$ is to use objective functions which are weighted averages of the compliance for a number of independent loading cases. That is, if $N$ independent loading cases are to be considered, the weighted compliance objective function would be

$\Pi\left(\mathbf{b}, \mathbf{u}^{(1)}, \mathbf{u}^{(2)}, \ldots, \mathbf{u}^{(N)}\right)=\sum_{n=1}^{N} \beta_{n} \Pi^{(n)}\left(\mathbf{b}, \mathbf{u}^{(n)}\right)$,

where the subscript or superscript $(n)$ represents the load case number; $\beta_{(n)}$ a constant weighting factor for each load case; $\pi^{(n)}$ the $n$th compliance functional value; and $\mathbf{u}^{(n)}$ the displacement field for the $n$th loading/restraint case.

In gradient based [1st order] optimization algorithms, it is essential that the total design gradient of the objective and constraint functionals be accurately and efficiently computable. Thus for weighted compliance functionals, we must be able to compute:

$$
\begin{aligned}
& \frac{\mathrm{d} \Pi\left(\mathbf{b}, \mathbf{u}^{(1)}, \mathbf{u}^{(2)}, \ldots, \mathbf{u}^{(N)}\right)}{\mathrm{d} \mathbf{b}} \\
& \quad=\sum_{n=1}^{N} \beta_{n}\left[\frac{\partial \Pi^{(n)}}{\partial \mathbf{b}}+\frac{\partial \Pi^{(n)}}{\partial \mathbf{u}^{(n)}} \frac{\mathrm{d} \mathbf{u}^{(n)}}{d \mathbf{b}}\right] .
\end{aligned}
$$

Using adjoint sensitivity analysis methods [28] these are directly computed as

$\frac{\mathrm{d} \Pi}{\mathrm{d} \mathbf{b}}=\sum_{n=1}^{N} \beta_{n}\left[\frac{\partial \Pi^{(n)}}{\partial \mathbf{b}}+\mathbf{u}^{a(n)} \cdot \frac{\partial \mathbf{r}^{(n)}}{\partial \mathbf{b}}\right]$

where $\mathbf{u}^{\mathrm{a}(n)}$ is the 'adjoint displacement field' for the $n$th loading case and is a solution of the $n$th 'adjoint problem'

$\mathbf{K}^{(n)} \cdot \mathbf{u}^{\mathrm{a}(n)}=-\frac{\partial \Pi^{(n)}}{\partial \mathbf{u}^{(n)}}$.

In those structural topology optimization problems, where the objective is to minimize the compliance of a structure subjected to a set of fixed external load cases $\mathbf{f}^{\operatorname{ext}(n)}, n \in\{1, \ldots, N\}$, the compliance functional (16) reduces to:

$\Pi=\sum_{n=1}^{N} \beta_{n}\left[\frac{1}{2} \mathbf{f}^{\operatorname{ext}(n)} \cdot \mathbf{u}^{(n)}\right]$, 
and the corresponding design gradient of the weighted compliance functional is simply

$\frac{\mathrm{d} \Pi}{\mathrm{d} \mathbf{b}}=-\frac{1}{2} \sum_{n=1}^{N} \beta_{n}\left[\int_{S} \epsilon^{(n)}: \frac{\partial \boldsymbol{\sigma}^{(n)}}{\partial \mathbf{b}} \mathrm{d} \Omega_{\mathrm{S}}\right]$,

where $\boldsymbol{\epsilon}^{(n)}$ and $\boldsymbol{\sigma}^{(n)}$ are the respective strain and stress fields due to the $n$th loading case. The quantity $\partial \boldsymbol{\sigma}^{(n)} /$ $\partial \mathbf{b}$ in Eq. (21) is the 'stress design gradient' and clearly depends upon the mixing rule being employed. The evaluation of this 'stress design gradient' for the hybrid Voigt-Reuss mixing rules was treated in Refs $[4,5]$.

\subsubsection{Eigenmode analysis}

Free vibrational modes of linear elastic structures are characterized by the eigenvalue equation

$\mathbf{0}=\left(\mathbf{K}-\lambda_{l} \mathbf{M}\right) \mathbf{y}_{l}$,

where $\mathbf{K}$ is the structural stiffness matrix, $\mathbf{M}$ the mass matrix, $\lambda_{l}$ the $l$ th vibrational eigenvalue, and $\mathbf{y}_{l}$ the corresponding eigenvector which describes the $l$ th mode of vibration. Since each element of $\Omega_{\mathrm{S}}$ generally contains a mixture of materials, the elastic constitutive tensor $\mathbf{C}$ at the element level used in computing $\mathbf{K}$ is provided by the mixing rules in Section 2.2, while the local density of the mixture in each element used in computing $\mathbf{M}$ is given by the relation

$\rho=\phi_{\mathscr{A}} \rho_{\mathscr{A}}+\phi_{\mathscr{B}} \rho_{\mathscr{B}}$.

For solid-void applications as treated in this paper, the density of the void phase $\rho_{\text {void }}$ is theoretically zero, but a small density compared to that of the solid phase $\rho_{\text {solid }}$ is maintained to avoid singularity of the eigenvalue Eq. (22). The density of the void material $\mathscr{B}$ is here taken as $\rho_{\text {void }}=10^{-6} \rho_{\text {solid }}$.

\subsubsection{Eigenvalue functional}

One method for optimizing the overall stiffness of a structure without reference to any specific loadings is to maximize a functional $\mathscr{L}$ which is a linear combination of the first $J$ vibrational eigenvalues:

$\mathscr{L}=\sum_{k=1}^{J} \beta_{k} \lambda_{k}$

where the $\lambda$ s are vibrational eigenvalues and the $\beta$ s are non-negative constant weighting factors. When $\mathscr{L}$ contains only simple nonrepeated eigenvalues, first order design sensitivity analysis is quite straightforward since

$\frac{\mathrm{d} \mathscr{L}}{\mathrm{d} \mathbf{b}}=\sum_{k=1}^{K} \beta_{k} \frac{\mathrm{d} \lambda_{k}}{\mathrm{~d} \mathbf{b}}$ where

$\frac{\mathrm{d} \lambda_{k}}{\mathrm{~d} \mathbf{b}}=\frac{\mathbf{y}_{k} \cdot\left(\frac{\partial \mathbf{K}}{\partial \mathbf{b}}-\lambda_{k} \frac{\partial \mathbf{M}}{\partial \mathbf{b}}\right) \cdot \mathbf{y}_{k}}{\mathbf{y}_{k} \cdot \mathbf{M} \cdot \mathbf{y}_{k}}$.

When $\mathscr{L}$ contains nonsimple, repeated eigenvalues, sensitivity analysis for the repeated roots can be somewhat more complicated. Unless other precautions are taken, the procedures suggested in Refs $[19,20]$ for repeated roots are usually required. However, for those classes of problems where nonsimple vibrational eigenvalues occur due strictly to the symmetry of the structure, then the symmetry reduction methods of Section 3 can be employed to alleviate the difficulty and design sensitivity analysis of functionals containing repeated eigenvalues can proceed along the lines of Eqs. (25) and (26) without additional complications or precautions. This is discussed in more detail in Section 4.

\section{A symmetry reduction method}

\subsection{Motivation}

There are two primary reasons for imposing geometrical symmetry on material layout designs. First, even when the design loads are asymmetrical the designer may still seek a symmetric design so that reversed loading cases can be accommodated equally well. Second, even when both the design loads and boundary conditions applied to $\Omega_{\mathrm{S}}$ are symmetrical, the resulting material layout field $\mathbf{b}$ as obtained through standard optimization techniques, will not necessarily feature the expected geometrical symmetries. While one may start with a symmetrical design field $\mathbf{b}$, small numerical perturbations from symmetry will arise naturally due to roundoff errors in the optimization and design sensitivity analysis procedures. These asymmetry perturbations may continue to grow resulting in a highly asymmetric design, or they may remain relatively small. The intent here is not to analyze the stability of these perturbations, but to present a robust strategy for imposing spatial symmetry on material layout design $\mathbf{b}$.

There are some special cases where symmetrical design solutions can be achieved simply by using symmetry (if it exists) in the analysis problem. If strict symmetry is known to exist in the analysis problems being considered for design optimization (i.e. loadings and/or free vibrations), then one can work with a subset of the physical design space as opposed to the full domain, with considerable savings in computational effort. However, such symmetries often times do not exist, and the analyst/designer is forced to work with 


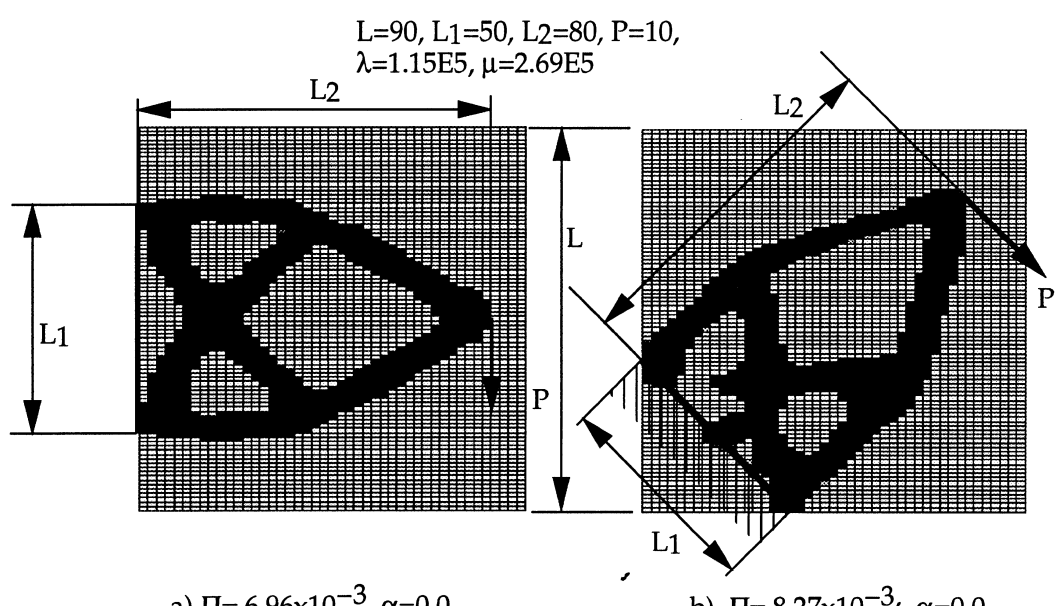

a) $\Pi=6.96 \times 10^{-3}, \alpha=0.0$

b) $\Pi=8.27 \times 10^{-3}, \alpha=0.0$

Fig. 2. The aligned cantilever design problem (a) and solutions obtained; the mis-aligned cantilever problem (b) with an asymmetrical design solution.

the entire structural domain. Since this is the more general and common case, the method developed below uses the full structural domain, and imposes either gross or exact symmetry on the design, depending upon the symmetry of the mesh discretization.

To demonstrate the need for symmetry control methods, the short cantilever topology design problem is considered here. The cantilever beam design problem (Fig. 2a) has been used extensively as a test problem by a number of investigators, as for example in Refs [1, 3, 4, 11,29-31], with the mis-aligned version of this problem considered to be especially challenging problem. Here, we solve this as a compliance minimization problem with a $25 \%$ global solid volume fraction constraint for both an aligned mesh, and a mis-aligned mesh consisting of $(45 \times 90)$ rectangular bilinear continuum finite elements each having an aspect ratio of $2: 1$. All calculations of this problem used filtering with the default filter parameters as described in [4]. Fig. 2a shows the pure Reuss solution $(\alpha=0)$ for the aligned mesh problem without symmetry control. Even without symmetry control, the design is roughly symmetric, as one would expect, due to both the symmetry of the mesh and the material response with respect to the applied load. However, when the problem is solved so that the load and restraints are not aligned with the mesh, then the asymmetrical design obtained in Fig. $2 b$ is obtained. The procedure presented below efficiently solves this problem of asymmetrical material layout designs.

\subsection{Symmetry by design space reduction}

3.2.1. Basic procedure of the symmetry reduction method

One can imagine the structural domain $\Omega_{\mathrm{S}}$ as being cut by one or more desired symmetry planes $(\mathbf{a} \cdot \mathbf{X}-$ $c=0)$. Whereas the structural domain $\Omega_{\mathrm{S}}$ should be grossly (but not necessarily exactly) symmetrical about these planes, the loading/boundary conditions on $\Omega_{\mathrm{S}}$ need not be at all symmetrical with respect to the specified plane(s). Furthermore, the mesh discretization need not be precisely symmetrical with respect to the symmetry planes. The proposed symmetry enforcement method reduces the dimension of design vector $\mathbf{b}$ space by taking design variables from the elements that lie on one side of the plane $(\mathbf{a} \cdot \mathbf{X}-c>0)$ and merging them with the design variables in the corresponding elements that lie on the other side of the symmetry plane $(\mathbf{a} \cdot \mathbf{X}-c<0)$. The typical $i$ th finite element can be characterized by its centroidal coordinates $\mathbf{X}_{i c}$. Two elements, say the $i$ th and $j$ th elements, are corresponding image element with respect to the symmetry plane (Fig. 3a) if:

1. They are on opposite sides of the plane.

2. They have the same linear distances to the symmetry plane.

3. They project to the same point $\mathbf{X}_{c}^{*}$ on the symmetry plane.

Since in reality, no two elements will have identical projections or distances to the symmetry plane, finding corresponding image elements on opposite sides of the symmetry plane is not a matter of finding an exact corresponding image element, but rather of finding the closest image element within certain tolerances. The algorithm used by the authors to find corresponding image elements is shown in Box 1.

If a symmetry plane is not defined the volume fraction of each element represents its own design variable as shown in Fig. 3b, but if symmetry reduction is employed, then design variables for corresponding image elements are unified and the design variable vector is condensed as shown in Fig. 3c. That is, volume 
Initialization Phase

$$
\begin{array}{ll}
\text { compute } r_{\max }=\left\|\mathbf{X}_{\max }-\mathbf{X}_{\min }\right\| & : \text { length scale of mesh } \\
\text { define } \epsilon_{1} \text { and } \epsilon_{2} & : \text { two small non-dimensional parameters }
\end{array}
$$

For each symmetry plane. . .

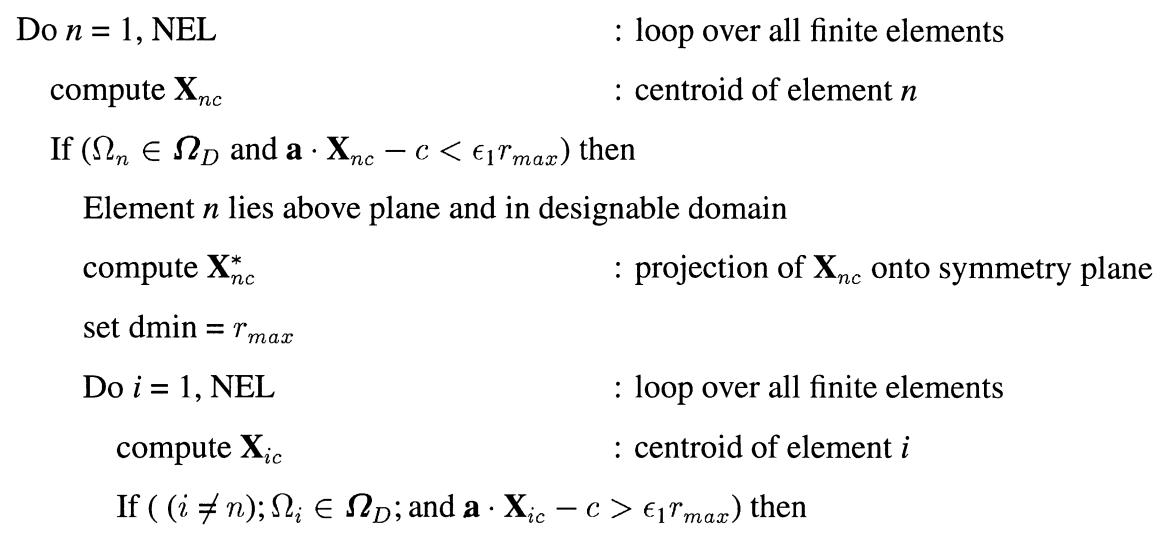

Element $i$ is below the plane and in designable domain

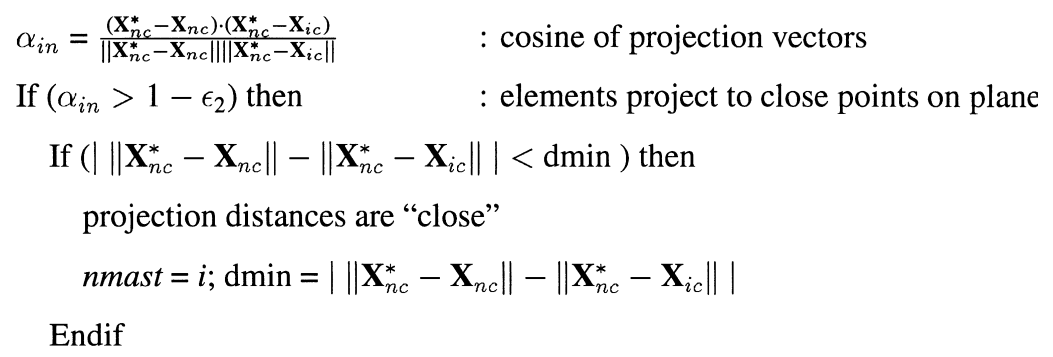

Endif

Endif

Enddo

Endif

Merge the design variables of element $n$ and image element nmast.

Enddo

Box 1. Algorithm for identifying corresponding image elements.

fractions of corresponding image elements $\phi_{i}$ and $\phi_{j}$ are made to share the design variable $\mathrm{b}_{k}^{\mathrm{r}}$ in the reduced design vector $\mathbf{b}^{\mathrm{r}}$, as shown in Fig. 3c. The vector $\mathbf{b}^{\mathbf{r}}$ here is thus defined as a reduced design vector since it contains fewer design variables than $\mathbf{b}$. The number of elements (NEL), the number of design variables (NDV), and the number of non-designable elements (NELN), thus, have the following relations:

$\mathrm{NDV}=\mathrm{NEL}-\mathrm{NELN}$; no symmetry reduction

$\mathrm{NDV} \leq \mathrm{NEL}-\mathrm{NELN} ;$ symmetry reduction.
This geometrically based symmetry reduction procedure is extremely easy to program and can be applied recursively with an arbitrary number of symmetry planes as is demonstrated in the solved problems of Section 4. Each time an additional symmetry plane is introduced, the dimension of the design vector $\mathbf{b}$ is effectively halved. While this method is similar in spirit to symmetry reduction methods like nodal enslavements used in finite element analysis, it is geometrically based therefore and works effectively with both regular and irregular meshes which may or may not be sym- 


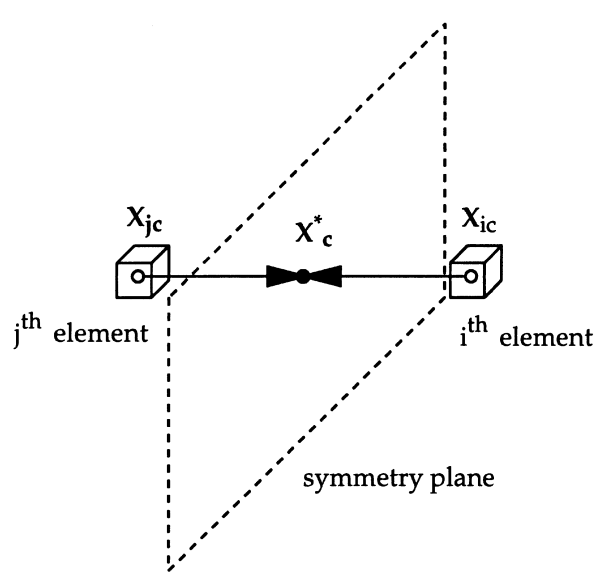

(a)

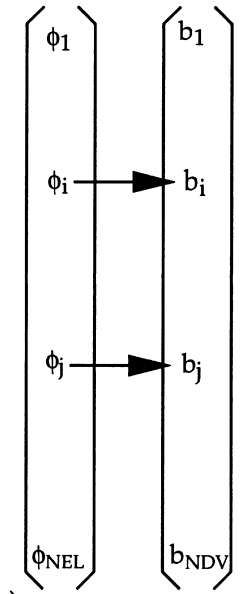

(b)

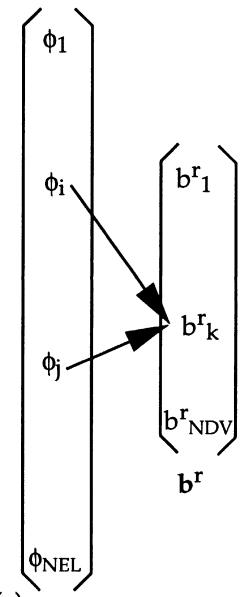

(c)

Fig. 3. (a) Schematic of corresponding elements with respect to a symmetry plane; (b) design mapping between element volume fractions and design variables without symmetry control; (c) design mapping between element volume fractions and design variables with symmetry control.

metric about the symmetry planes. Before topology optimization begins, a mapping vector of length NEL is generated which designates the relationship between individual finite element volume fractions and design variables in b. While enforcing symmetry and significantly reducing the size of the optimization problem, it has the added benefit of facilitating design sensitivity analysis in some pathological cases such as that of repeated vibrational eigenvalues in symmetrical structures, which will be discussed in Section 4.

Using the sensitivity expression (21) for forcecontrolled structures, the design sensitivity expression with the proposed symmetry reduction method is

$$
\begin{aligned}
\frac{\mathrm{d} \Pi^{\mathrm{B}}}{\mathrm{d} \mathbf{b}^{\mathrm{r}}} & =-\frac{1}{2} \int_{S} \boldsymbol{\epsilon}: \frac{\partial \boldsymbol{\sigma}}{\partial \mathbf{b}^{\mathrm{r}}} \mathrm{d} \Omega_{\mathrm{S}} \\
& =\sum_{n=1}^{\mathrm{NEL}}\left(-\frac{1}{2} \int_{n}\left[\boldsymbol{\epsilon}: \frac{\partial \boldsymbol{\sigma}}{\partial \phi_{n}} \frac{\partial \phi_{n}}{\partial \mathbf{b}^{\mathrm{r}}}\right] \mathrm{d} \Omega_{n}\right) .
\end{aligned}
$$

where $\partial \phi_{n} / \partial \mathbf{b}^{\mathrm{r}}$ is a design mapping term.

Attention is confined here to applying the symmetry reduction method to micro-structure free topology formulations which use scalar density or volume fraction type design variables. Application of the symmetry reduction method to relaxed topology design formulations that use microstructural design variables is also possible, although somewhat more complicated.

To demonstrate the efficacy of the proposed symmetry reduction method, the mis-aligned version of the short cantilever beam design problem is solved here again using a single symmetry plane. The solutions to this problem without and with a symmetry plane are shown in Fig. 4. Even though the finite element mesh employed here is not itself symmetrical with respect to the imposed symmetry plane, gross symmetry can still be successfully imposed on the topology design using the proposed symmetry reduction method.

The preceding example is one where a load was applied to the structural domain $\Omega_{\mathrm{S}}$, and due to the symmetry of the load with respect to the material response and the applied restraint conditions, one expects to achieve a symmetrical design solution. There are cases, however, where the loading system is not symmetrical with respect to the applied restraint conditions and yet symmetrical designs are desired to accommodate reversed loading conditions. An example of such a situation is the automotive deck lid problem which has previously been presented in the research literature $[4,32]$. Fig. 5 shows the full structural domain $\Omega_{\mathrm{S}}$, the boundary and loading conditions, and the design domain $\Omega_{\mathrm{D}}$ for the deck lid problem. The white region in the structure represents the design domain $\Omega_{\mathrm{D}}$ and the nondesignable black region $\Omega_{\mathrm{N}}$ contains solid material. A thin shell structure such as the deck lid uses both bending and membrane action to carry the applied torsional loads. In the topology optimization calculations performed for this problem, continuum degenerated shell elements were employed with reduced integration of both transverse shear and membrane stresses to avoid numerical locking behavior. For the fixed loads and restraints shown in Fig. 5, the topology design problem was formulated to minimize the elastic compliance of the deck lid under the applied loading with an upper bound on the global 


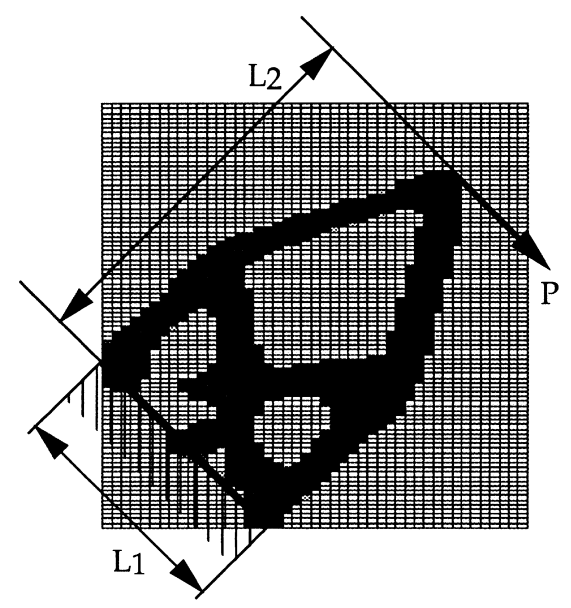

a) $\Pi=8.27 \times 10^{-3,} \alpha=0.0$

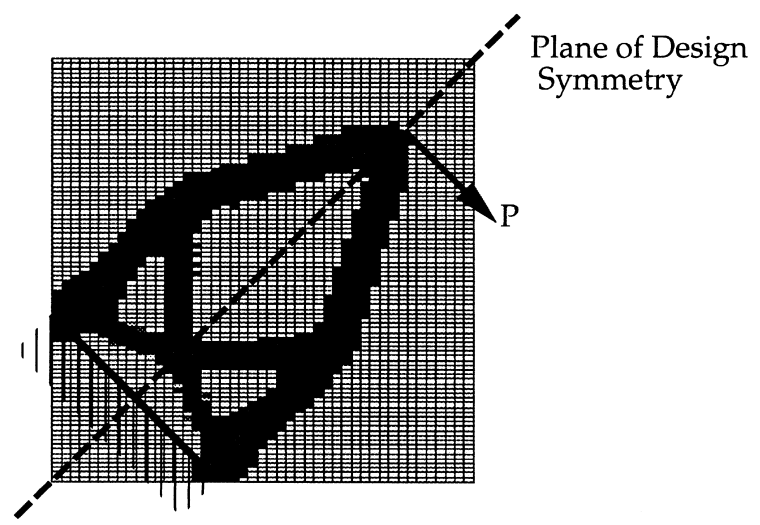

b) $\Pi=7.75 \times 10^{-3}, \alpha=0.0$, With symmetry control

Fig. 4. The mis-aligned cantilever design problem and solutions obtained without (a) and with (b) symmetry control.
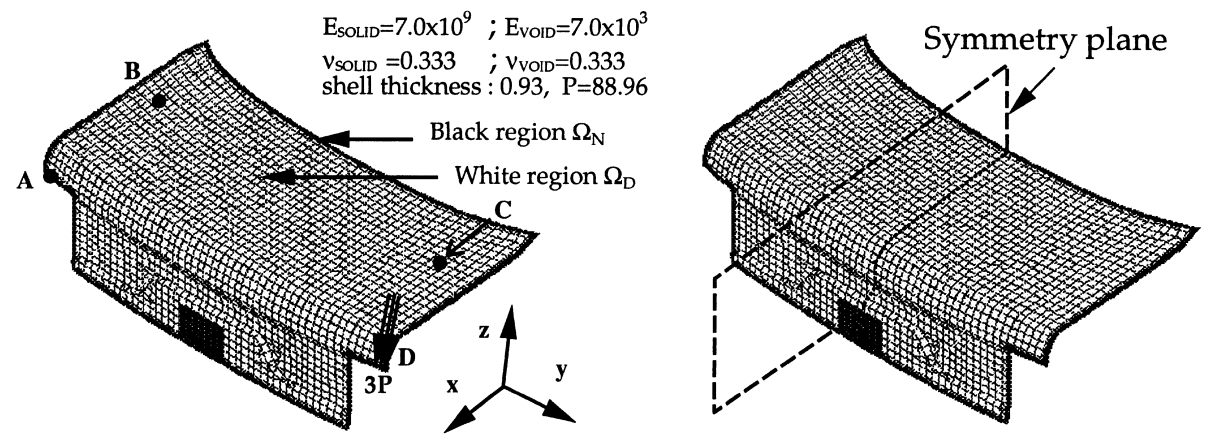

$$
\begin{aligned}
& \text { Boundary and Loading Conditions: } \\
& \text { A : restrained in z-direction } \\
& \text { B, C: all DOF's restrained except rotation } \\
& \text { D : about y-axis }
\end{aligned}
$$

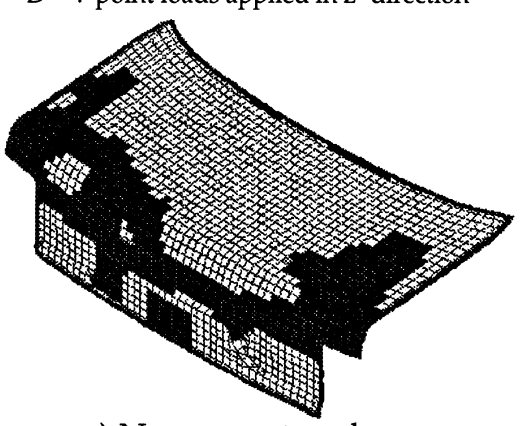

a) No symmetry planes

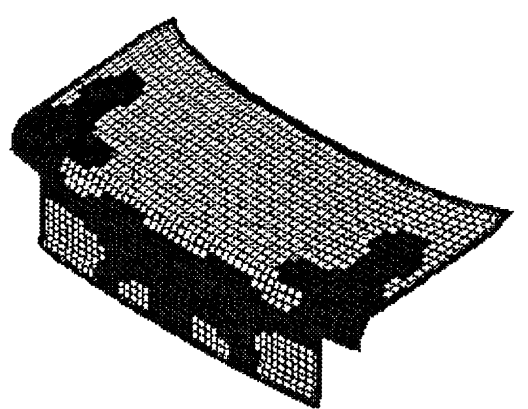

b) 1 symmetry plane $\Pi=23.20,89$ iterations

Fig. 5. Design domain and boundary/loading conditions for the deck lid problem along with solutions obtained (a) without and (b) with symmetry control. Initial design state: $\phi_{\text {solid }}=1.0$ on $\Omega_{\mathrm{D}}$. 
solid volume fraction: $\left\langle\phi_{\text {solid }}\right\rangle \leqslant 0.35$. The boundary and loading conditions applied to the deck are clearly asymmetrical and so one would expect an unsymmetrical design such as that shown in Fig. 5a if symmetry is not imposed on the layout. To obtain the more desirable symmetric layout shown in Fig. 5b which can accommodate a reversed loading equally well (structure loaded at A and restrained at D), a single symmetry plane was employed, which also halved the size of the optimization problem.

\section{Symmetry reduction and vibrational eigenvalues}

\subsection{Motivation}

Many structures whose structural stiffnesses and mass distributions are the same and symmetrical in two or more orthogonal directions invariably encounter the phenomenon of repeated, non-simple vibrational eigenvalues. It is recognized that the design sensitivity analysis of repeated eigenvalues is difficult due to the fact that they are generally not continuously differentiable, but are only directionally differentiable [17]. Accordingly, design sensitivities for repeated vibrational eigenvalues cannot be determined by expressions such as Eq. (26) unless special precautions are taken. In this section it will be shown that by applying symmetry reduction, the design gradients of many non-simple repeated eigenvalues can in fact be evaluated by Eq. (26).

As an example of a very simple and symmetrical structure that will encounter repeated vibrational eigenvalues, we consider a thin, solid flat rectangular plate which is pin supported along its edges as shown in Fig. 6. Such a plate is assumed to be governed by Kirchoff's plate theory, and so its vibrational eigenfrequencies are given by the expression [33] $\omega_{(m, n)}=\left(\frac{\pi^{4} D}{\rho h a^{4}}\right)^{1 / 2}\left[m^{2}+n^{2}\left(\frac{a}{b}\right)^{2}\right]$

where $a, b$ and $h$ are the dimensions of the plate in the $x, y$ and $z$ directions; $D=E h^{3} / 12\left(1-v^{2}\right)$ is the flexural stiffness of the plate, and $m, n$ are integer indices. The associated free vibrational spatial mode shapes of the plate are given by

$W_{(m, n)}(x, y)=C \sin \left(\frac{m \pi x}{a}\right) \sin \left(\frac{n \pi y}{b}\right)$.

Clearly, when the dimensions $a$ and $b$ of the plate are the same in the $x$ and $y$ directions, the plate will have an infinite sequence of repeated non-simple eigenvalues (i.e. $\left.\omega_{(m, n)}=\omega_{(n, m)} \forall m \neq n\right)$. None of these eigenvalues will be differentiable by Eq. (26) since the repeated eigenvalues do not have unique associated eigenmodes. The objective here is to demonstrate, however, that if the design space is sufficiently reduced using the proposed symmetry enforcement methods, then all of these repeated eigenvalues will indeed be fully differentiable by Eq. (26). This issue is discussed here in the context of this flat plate example, but the fundamental idea extends to much more general classes of structures as well.

\subsection{Differentiability and directional derivative}

A design sensitivity expression for simple, nonrepeated eigenvalues was shown in Eq. (26), but if the eigenvectors are M-orthonormalized, this expression can be re-written in simplified form as

$\frac{\mathrm{d} \lambda_{k}}{\mathrm{~d} \mathbf{b}}=\mathbf{y}_{k} \cdot\left(\frac{\partial \mathbf{K}}{\partial \mathbf{b}}-\lambda_{k} \frac{\partial \mathbf{M}}{\partial \mathbf{b}}\right) \cdot \mathbf{y}_{k}$

where it is reasonably assumed that the stiffness and mass matrices are continuously differentiable with

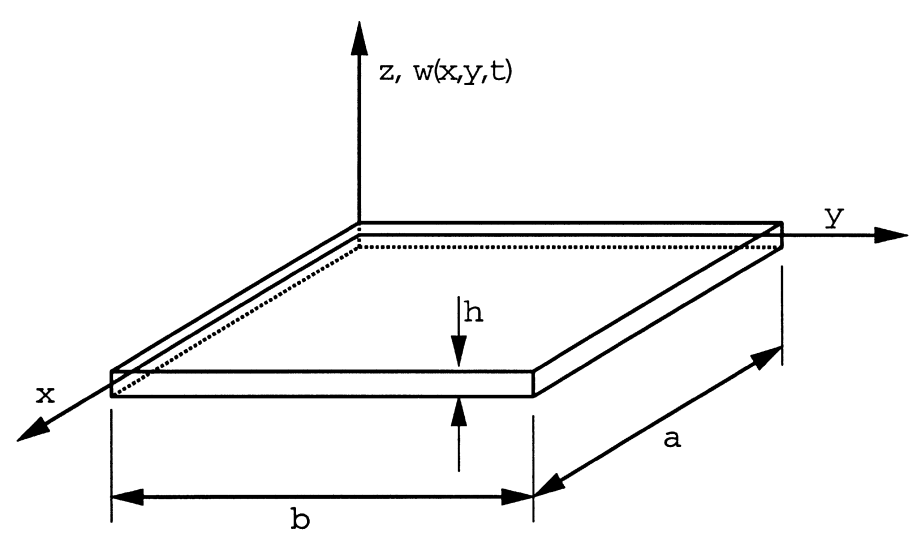

Fig. 6. Square flat plate which is pin supported along its edge boundaries. 
respect to designs $\mathbf{b}$. If the $k$ th eigenvalue is a nonsimple, repeated root of Eq. (22), then the design gradient given by Eq. (30) will have multiple vector-values in accordance with the multiple eigenvectors used to evaluate it. For this reason, non-simple, repeated eigenvalues are not continuously differentiable and are only directionally differentiable. In general, Eq. (30) is thus valid only for simple, non-repeated eigenvalues. This is discussed with simple examples in Ref. [34] with additional differentiability and continuity theorems for eigenvalue problems presented in Refs $[35,36]$.

To further investigate the differentiability of repeated eigenvalues, it is assumed that at a given design b there exists a non-simple eigenvalue $\lambda^{*}$ with multiplicity $s>1$. Following Ref. [34], the directional derivatives $\left(\lambda^{*}\right)_{k}^{\prime}$ of $\lambda^{*}$ with respect to the typical $k$ th design variable can be evaluated as the eigenvalues of the following auxiliary $s \times s$ matrix:

$\mathscr{A}_{i j}^{k}=\left[\mathbf{y}_{i} \cdot\left(\frac{\partial \mathbf{K}}{\partial b_{k}} \delta b_{k}-\lambda^{*} \frac{\partial \mathbf{M}}{\partial b_{k}} \delta b_{k}\right) \cdot \mathbf{y}_{j}\right] \quad i, j=1,2, \ldots, s$

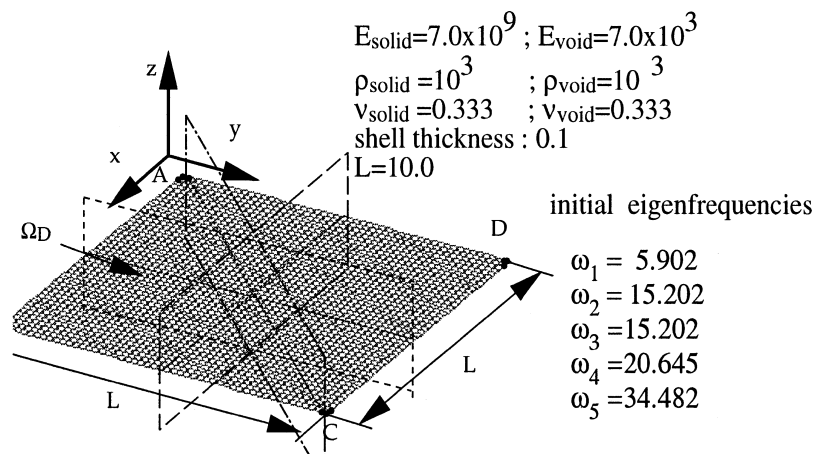

Boundary and Loading Conditions:

A,B,C,D pin supported. Three design

symmetry planes used are shown.

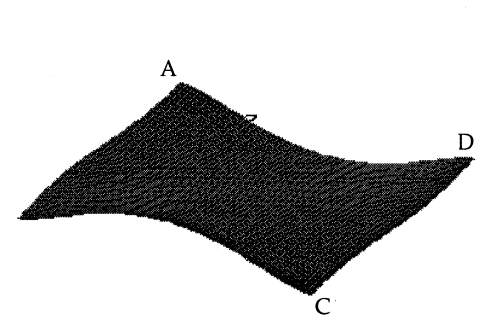

(c) 3rd eigenmode for solid plate where $\mathbf{y}_{i}$ and $\mathbf{y}_{j}(i, j=1,2, \ldots, s)$ are any M-orthonormal basis of the eigenvectors associated with $\lambda *(\mathbf{b})$. The eigenvalues of $\mathscr{A}_{i j}^{k}$ clearly depend on the direction of $\delta b_{k}$, as will the directional derivative $\lambda(*)_{k}^{\prime}$. To observe how the directional derivatives are related to design variations $\delta b_{k}$ the case of a non-simple eigenvalue $\lambda^{*}$ of multiplicity $s=2$ can be considered. The characteristic equation whose roots provide the eigenvalues of $\mathscr{A}^{k}$, Eq. (31) is simply

$$
\begin{aligned}
\left|\begin{array}{cc}
\mathscr{A}_{11}^{k}-\lambda^{\prime} & \mathscr{A}_{12}^{k} \\
\mathscr{A}_{21}^{k} & \mathscr{A}_{22}^{k}-\lambda^{\prime}
\end{array}\right|= & \mathscr{A}_{11}^{k} \mathscr{A}_{22}^{k}-\left(\mathscr{A}_{12}^{k}\right)^{2} \\
& -\left(\mathscr{A}_{11}^{k}+\mathscr{A}_{22}^{k}\right) \lambda^{\prime}+\left(\lambda^{\prime}\right)^{2}=0,
\end{aligned}
$$

where the fact that $\mathscr{A}_{12}^{k}=\mathscr{A}_{21}^{k}$ has been employed. If $\mathscr{A}_{12}^{k}$ is zero, the eigenvalues of $\mathscr{A}^{k}$ which are the directional derivatives $\left(\lambda^{*}\right)_{k}^{\prime}$ can be trivially obtained as $\left(\lambda^{*}\right)_{k}^{\prime}=\mathscr{A}_{11}^{k}, \mathscr{A}_{22}^{k}$, and $\left(\lambda^{*}\right)_{k}^{\prime}$ is clearly linear in $\delta b_{k}$. However, if $\mathscr{A}_{12}^{k}$ does not vanish, the quadratic formula must be used to find the roots of Eq. (31) as follows:

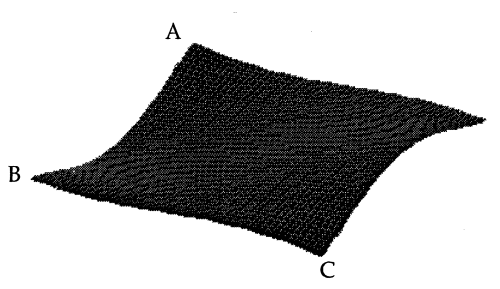

(b) 2nd eigenmode for solid plate

$$
\begin{aligned}
& \mathrm{L}=10.856 \\
& \omega_{\mathrm{i}}: \text { optimized eigenfrequencies } \\
& \beta_{\mathrm{i}} \text { : weighting factors } \\
& \omega_{1}=4.369 \quad \beta_{1}=2.87 \times 10^{-2} \\
& \omega_{2}=17.097 \quad \beta_{2}=4.33 \times 10^{-3} \\
& \omega_{3}=17.097 \quad \beta_{3}=4.33 \times 10^{-3} \quad \mathrm{~B} \\
& \omega_{4}=28.842 \quad \beta_{4}=2.35 \times 10^{-3} \\
& \omega_{5}=40.025 \quad \beta_{5}=8.41 \times 10^{-4}
\end{aligned}
$$

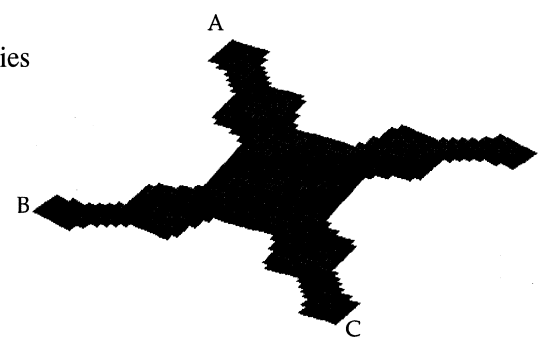

d) Optimized material layout toplogy with resulting natural frequencies.

Fig. 7. Square plate eigenvalue optimization problem. (a) Plate properties, restraint conditions, three design symmetry planes and natural frequencies before topology optimization; (b) second eigenmode for solid plate; (c) third eigenmode for solid plate; and (d) optimized plate topology (first eigenmode shown) with resulting first five eigenfrequencies. 


$$
\begin{aligned}
\lambda_{i, k}^{\prime}= & \frac{1}{2}\left\{\left(\mathscr{A}_{11}^{k}+\mathscr{A}_{22}^{k}\right)\right. \\
& \left. \pm \sqrt{\left(\mathscr{A}_{11}^{k}+\mathscr{A}_{22}^{k}\right)^{2}-4\left[\mathscr{A}_{11}^{k} \mathscr{A}_{22}^{k}-\left(\mathscr{A}_{12}^{k}\right)^{2}\right]}\right\}, \\
i= & 1,2 .
\end{aligned}
$$

Due to the square root term, the roots as expressed by Eq. (33) are generally not linear in $\delta b_{k}$. Thus, unless $\mathscr{A}_{12}^{k}$ vanishes, $\left(\lambda^{*}\right)_{k}^{\prime}$ is not linear in $\delta b_{k}$ and the non-simple, repeated eigenvalues will not be fully differentiable but only directionally differentiable.

\subsection{Symmetry reduction and repeated eigenvalues}

To address the difficulties in differentiating repeated eigenvalues, the following useful proposition will be considered in this section.

Proposition. In a symmetric structure, which has non-simple repeated eigenvalues as by Eq. (28), when the design space is reduced by the proposed symmetry reduction methods of Section 3, the auxiliary directional derivative matrix (31) is diagonal for all design variations. [Specifically, $\mathscr{A}_{i j}^{k}=0, \forall k \in\{1, \ldots, N D V\}$ and $\forall i \neq j,(i, j=1, \ldots, s)$.] Non-simple repeated eigenvalues are thus fully differentiable with respect to the reduced design variables, and design sensitivities can thus be computed directly by Eq. (30).

To show that this is true, consider that for a nonsimple repeated eigenvalue $\lambda^{*}$ of multiplicity $s$, the auxiliary matrix to solve for the directional design derivatives can be written as

$$
\begin{aligned}
\mathscr{A}_{i j}^{k} & =\mathbf{y}_{i}^{*} \cdot\left[\int_{s} \mathbf{B}^{\mathrm{T}}\left(\frac{\partial \mathbf{C}}{\partial b_{k}^{\mathrm{r}}} \delta b_{k}^{\mathrm{r}}\right) \mathbf{B} \mathrm{d} \Omega_{\mathrm{S}}\right. \\
& \left.-\lambda^{*} \int_{s}\left(\frac{\partial \rho}{\partial b_{k}^{\mathrm{r}}} \delta b_{k}^{\mathrm{r}}\right) \mathbf{N} \mathbf{N} \mathrm{d} \Omega_{S}\right] \cdot \mathbf{y}_{j}^{*} \\
& =\sum_{n=1}^{N E L} \mathbf{y}_{i n}^{*} \cdot\left[\int_{n} \mathbf{B}^{\mathrm{T}}\left(\frac{\partial \mathbf{C}}{\partial \phi_{n}} \frac{\partial \phi_{n}}{\partial b_{k}^{\mathrm{r}}} \delta b_{k}^{\mathrm{r}}\right) \mathbf{B} \mathrm{d} \Omega_{n}\right. \\
& \left.-\lambda^{*} \int_{n}\left(\frac{\partial \rho}{\partial \phi_{n}} \frac{\partial \phi_{n}}{\partial b_{k}^{\mathrm{r}}} \delta b_{k}^{\mathrm{r}}\right) \mathbf{N N} \mathrm{d} \Omega_{n}\right] \cdot \mathbf{y}_{j n}^{*}
\end{aligned}
$$

where $k \in\{1, \ldots, N D V\}, \mathbf{y}_{i}^{*}$ and $\mathbf{y}_{j}^{*}(i, j=1,2, \ldots, s)$ form the $\mathbf{M}$-orthonormal basis of the eigenvectors associated with the non-simple eigenvalue $\lambda^{*}$, and $\mathbf{y}_{\text {in }}^{*}$ and $\mathbf{y}_{j n}^{*}$ are localized eigenvectors of $\mathbf{y}_{i}^{*}$ and $\mathbf{y}_{j}^{*}$ for $n$th element.

For simplicity and the sake of a specific example, it will be assumed that we are dealing with the solid thin square plate of Section 4.1 whose design space has

\footnotetext{
${ }^{2}$ The details are omitted here but are presented at length in Ref. [15].
}

been reduced by employing three symmetry planes, as shown in Fig. 7. The second vibrational eigenvalue for this plate is non-simple and repeated, and has the following eigenmodes:

$\mathbf{y}_{1}^{*}=\sin \left(\frac{2 \pi x}{a}\right) \sin \left(\frac{\pi y}{b}\right)$

$\mathbf{y}_{2}^{*}=\sin \left(\frac{\pi x}{a}\right) \sin \left(\frac{2 \pi y}{b}\right)$

These modes have both symmetrical and anti-symmetrical components about the center of the plate, and furthermore are identical except for the fact that they are rotated by $90^{\circ}$ about the $z$-axis. When these modes are inserted into Eq. (34), it can be shown that the off-diagonal terms $\mathscr{A}_{12}^{k}$ of the auxiliary matrix vanish and furthermore that $\mathscr{A}_{11}^{k}=\mathscr{A}_{22}^{k}{ }^{2}$ Accordingly, when the design space of symmetric structures is properly reduced, repeated vibrational eigenvalues of the type considered here can in fact be straightforwardly differentiated using Eq. (30). This greatly simplifies the problem of design sensitivity analysis of many repeated eigenvalues and thus facilitates continuum topology optimization of structures for maximal vibrational eigenvalues. In essence, with symmetry reduction, different eigenmodes associated with nonsimple, repeated eigenvalues are projected into a greatly reduced design space in which they appear identical.

\subsection{Demonstrative example}

The topology design optimization of material layout in a pin-supported square plate structural domain $\Omega_{\mathrm{S}}$ (Fig. 7) is considered here. Similar problems have been considered previously in Ref. [13] to minimize the elastic compliance in square plates under static loading conditions. Here, the Voigt-Reuss topology formulation is applied with the proposed symmetry reduction method to maximize a composite functional $\mathscr{L}=\Sigma_{k=1}^{5} \lambda_{k} \beta_{k}$ of the first five eigenvalues of the square plate, in which the $\lambda_{k}$ and $\beta_{k}$ are, respectively, the $k$ th eigenvalue and weighting factor. (Eigenvalues are related to eigenfrequencies by the relation $\lambda_{k}=\omega_{k}^{2}$.) Since the structural domain $\Omega_{\mathrm{S}}$ is square and symmetrically restrained, the second eigenvalue is non-simple with multiplicity $s=2$ as shown in Fig. 7b and c. This non-simple eigenvalue is thus treated in the objective function $\mathscr{L}$ as the second and third eigenvalues. The eigenvalue maximization problem was solved with material constraint $\left\langle\phi_{\text {solid }}\right\rangle \leqslant 0.50$ using an SLP optimization algorithm [37] with a move limit of $\Delta_{M}=0.05$. Spatial filtering [4] with default filter 
parameter values was also employed to preclude checkerboarding solutions. Since the proposed symmetry reduction method was employed, design sensitivities of the objective function were directly computed by Eq. (30). Fig. 7d shows the material layout obtained solution using a pure Reuss formulation $(\alpha=0)$ with the first eigenmode shown. The obtained material layout is optimal, discrete, symmetric, and stable.

\section{Summary and conclusions}

It has been hypothesized that asymmetrical material layout solutions are caused by the non-convexity of highly penalized continuum topology design formulations coupled with limited precision in numerical computations. In this study, a novel symmetry reduction method to control and stabilize non-convex topology design formulations has been investigated and demonstrated on simple, representative examples.

Benefits of the proposed symmetry reduction method are that it produces symmetrical material layout designs while dramatically reducing the size of the optimization problem that needs to be solved. In addition, the symmetry reduction method has been found to ease the difficulties in design sensitivity analysis of some, but not all, repeated vibrational eigenvalues. The symmetry reduction method is very simple and easy to implement, and can be applied with topology optimization of structures not just for linear elastic applications as considered here, but a wide variety of other structural topology applications including composite material design, strength optimization, compliant mechanism design, and viscoelastic damping optimization. In particular, for topology optimization of inelastic structural systems, design sensitivity analysis can be computationally intensive in proportion to the number of design variables [5]. Since the reduction techniques proposed here significantly reduce the number of design variables, and in some cases the number of analyses required as well, they can significantly reduce the computational effort required in topology optimization of inelastic structural systems.

\section{References}

[1] Suzuki K, Kikuchi N. A homogenization method for shape and topology optimization. Computer Methods in Applied Mechanics and Engineering 1991;93.

[2] Jog CS, Haber RB, Bendsoe MP. Topology design with optimized, self-adaptive materials. International Journal for Numerical Methods in Engineering 1994;37:1323-50.

[3] Sigmund O. Design of material structures using topology optimization, Report S69. Danish Center for Applied
Mathematics and Mechanics, Technical University of Denmark, Lyngby, Denmark, 1994.

[4] Swan CC, Kosaka I. Voigt-Reuss topology optimization for structures with linear elastic material behaviours. International Journal for Numerical Methods in Engineering 1997;40:3033-57.

[5] Swan CC, Kosaka I. Voigt-Reuss topology optimization for structures with nonlinear material behaviours. International Journal for Numerical Methods in Engineering 1997;40:3785-814.

[6] Swan CC, Arora JS. Topology design of material layout in structured composites of high stiffness and strength. Structural Optimization 1997;13:45-59.

[7] Kosaka I, Swan CC. Continuum topology optimization of viscoelastic systems of high stiffness and high damping. (to appear), 1998.

[8] Ananthasuresh GK, Kota S, Gianchandani Y. A methodical approach to design of compliant micromechanisms. In: Proceedings of Solid-state sensor and actuator workshop, 1994. p. 189-194.

[9] Sigmund O. On the design of compliant mechanisms using topology optimization. Mechanics of Structures and Machinery, 1997.

[10] Kosaka I, Swan CC. Design of compliant structural mechanisms with Voigt-Reuss topology optimization. (to appear), 1998.

[11] Diaz AR, Bendsoe MP. Shape optimization of structures for multiple loading conditions using a homogenization method. Structural Optimization 1992;4:17-22.

[12] Bendsoe MP. Optimal shape design as a material distribution problem. Structural Optimization 1989;1:193-202.

[13] Ramm E, Bletzinger KU, Reitinger R, Maute K. The challenge of structural optimization. In: Topping BHV, Papadrakakis M., editors. Advances in Structural Optimization. Edinburgh: Civil-Comp Press, 1994. p. 2753.

[14] Mlejnek HP, Schirrmacher R. An engineer's approach to optimal material distribution and shape finding. Computer Methods in Applied Mechanics and Engineering 1993;106:1-26.

[15] Kosaka I. A conceptual design method for structures and structural materials. Ph.D thesis, College of Engineering, The University of Iowa, 1997.

[16] Bendsoe MP, Diaz AR, Lipton R, Taylor JE. Optimal design of material properties and material distribution for multiple loading conditions. International Journal for Numerical Methods in Engineering 1995;38:1149-70.

[17] Haug EJ, Rousselet B. Design sensitivity analysis in structural mechanics. II. Eigenvalue variations. Journal of Structural Mechanics 1980;8(2):161-86.

[18] Choi KK, Haug EJ. Repeated eigenvalues in mechanical optimization problems. Contemporary Mathematics 1981;4:61-86.

[19] Choi KK, Haug EJ, Lam HL. A numerical method for distributed parameter structural optimization problems with repeated eigenvalues. Journal of Structural Mechanics 1982;10(2):191-207.

[20] Choi KK, Haug EJ, Seong HG. An iterative method for finite dimensional structural optimization problems with repeated eigenvalues. International Journal for Numerical Method in Engineering 1983;19:93-112. 
[21] Olhoff N, Rasmussen SH. On single and bimodal optimum buckling loads of clamped columns. International Journal of Solids and Structures 1977;13:606-14.

[22] Ojalvo IU. Efficient computation of modal sensitivities for system with repeated frequencies. AIAA Journal 1988;4:361-6.

[23] Chen TY. Design sensitivity analysis for repeated eigenvalues in structural design. AIAA Journal 1993;4:2347-50.

[24] Diaz A, Sigmund O. Checkerboard patterns in layout optimization. Structural Optimization 1995;10:40-5.

[25] Jog CS, Haber RB. Stability of finite element models for distributed-parameter optimization and topology design. Computer Methods in Applied Mechanics and Engineering 1996;130:203-26.

[26] Haber RB, Jog CS, Bendsoe MP. A new approach to variable-topology shape design using a constraint on perimeter. Structural Optimization 1996;11:1-12.

[27] Ma Z-D, Kikuchi N, Cheng H-C, Hagiwara I. Topological optimization technique for free vibration problems. Journal of Applied Mechanics 1995;62:200-7.

[28] Arora JS, Cardoso JB. Variational principle for shape design sensitivity analysis. AIAA Journal 1992;30:538-47.

[29] Yang RJ, Chuang CH. Optimal topology design using linear programming. Computers \& Structures 1994;52$2: 265-75$.
[30] Allaire G, Kohn RV. Topology optimal design and optimal shape design using homogenization. In: Bendsoe MP, Mota Soares CA, editors. Topology design of structures. Boston, MA: Kluwer Academic, 1993. p. 207-218.

[31] Bendsoe MP, Diaz A, Kikuchi N. Topology and generalized layout optimization of elastic structures. In: Bendsoe MP, Mota Soares CA, editors. Topology design of structures. Boston, MA: Kluwer Academic, 1993. p. 159-205.

[32] Chirehdast M, Sankaranarayanan S, Ambo SD, Johanson RP. Validation of topology optimization for component design. SDM Preprint, 1994.

[33] Leissa AW. Vibration of Plates. NASA, SP-160, National Aeronautics and Space Administration, Washington, DC, 1969.

[34] Haug EJ, Choi KK, Komkov V. Design sensitivity analysis of structural systems. London: Academic Press, 1986.

[35] Kato T. Perturbation theory for linear operators. Berlin: Springer, 1976.

[36] Vainberg MM. Variational method and methods of monotone operators in the theory of nonlinear equations. Halsted Press, Wiley, 1973.

[37] Mijar AR. Topology design of structures using SLP. M.S. thesis, College of Engineering, The University of Iowa, Iowa City, 1997. 